Article

\title{
Increasing Surface Plasmons Propagation via Photonic Nanojets with Periodically Spaced 3D Dielectric Cuboids
}

\author{
Victor Pacheco-Peña ${ }^{1}$, Igor V. Minin ${ }^{2}$, Oleg V. Minin ${ }^{2}$ and Miguel Beruete ${ }^{1,3, *}$ \\ 1 Antennas Group-TERALAB, Public University of Navarra, Campus de Arrosadía, 31006 Pamplona, Spain; \\ victor.pacheco@unavarra.es \\ 2 National Research Tomsk State University, Lenina Ave. 36, Tomsk 634050, Russia; \\ prof.minin@gmail.com (I.V.M.); oleg.minin@ngs.ru (O.V.M.) \\ 3 Institute of Smart Cities, University of Navarra, Campus de Arrosadía, 31006 Pamplona, Spain \\ * Correspondence: miguel.beruete@unavarra.es; Tel.: +34-948-169-727
}

Received: 28 February 2016; Accepted: 17 March 2016; Published: 21 March 2016

\begin{abstract}
A structure based on periodically arranged 3D dielectric cuboids connected by photonic nanojets (PNJs) is proposed with the aim of increasing the propagation distance of surface plasmon polaritons (SPPs) at the telecom wavelength of $1550 \mathrm{~nm}$. The performance of the structure is evaluated and compared with the case without the cuboids demonstrating that the SPPs propagation length is enhanced by a factor greater than 2 , reaching a value of approximately $19 \lambda_{0}$, when the gap between the cuboids is $2.5 \lambda_{0}$. Also, the dependence of the propagation length with the height of the cubes is evaluated, showing that this parameter is critical for a good performance of the chain. A subwavelength resolution is obtained for all the jets generated at the output of the cuboids.
\end{abstract}

Keywords: photonic nanojets; surface plasmons; optical waveguide; dielectric waveguide; telecom wavelengths

\section{Introduction}

The intrinsic limit imposed by the diffraction of the electromagnetic waves has been intensively studied by the scientific community in order to improve the performance of microscopy applications [1]. Different techniques have been reported to surpass this limit, using for instance metamaterials [2,3], lenses [4-7] and microspherical particles [8,9].

Within this context, photonic nanojets (PNJs) were reported and experimentally demonstrated in the last decade with the aim to achieve a resolution below the diffraction limit [10-12]. A PNJ is a region with a strong electric field concentration just at the output surface of micro-scaled dielectric particles (typically spheres or cylinders) illuminated with a plane wave. It has been demonstrated that the ratio between the refractive index of the dielectric and the background medium should be $<2$ in order to excite a PNJs with a subwavelength focus [12]. Based on this, different configurations have been reported to improve the resolution of PNJs such as non-spherical particles [13], Luneburg lenses $[14,15]$ and multilayered elements [16]. Demonstrating that these structures can be used in applications such as nanoscopes and optical switching [8,17].

Recently, 3D dielectric cuboids have been proposed and experimentally demonstrated as an alternative structure to produce PNJs at sub-THz frequencies $[18,19]$. It has been demonstrated that the same refractive index ratio constraint applies to cuboids in order to produce jets at its output. Also 3D dielectric cuboids have been studied working in the reflection mode regime, demonstrating that the PNJs can also be excited with this configuration [20]. Additionally, it has been reported that this structure can be used as a waveguide by simply implementing a periodic array of 3D dielectric cuboids [21]. Interestingly, PNJs have also been proposed at optical wavelengths using surface plasmon 
polaritons (SPPs) instead of a plane wave as an excitation source [22,23], demonstrating that the location of the PNJ can be tailored by changing the height of the dielectric particles (cuboids or cylinders).

In this communication, we report a plasmonic waveguide consisting of periodically spaced 3D dielectric cuboids on top of a metal film excited by SPPs, able to almost double the propagation distance of SPPs at the telecommunication wavelength of $\lambda_{0}=1550 \mathrm{~nm}$ (which is a standard wavelength for optical applications). The structure is evaluated by periodically placing a total number of 6 cuboids and changing the gap between them. A study of the behavior when the height of the cuboids is changed from $80 \mathrm{~nm}$ to $240 \mathrm{~nm}$ is carried out for different distances between the cuboids, showing that the best performance in terms of the propagation distance of SPPs is achieved when the height of the cuboids is $120 \mathrm{~nm}$ and the gap between them is $2.5 \lambda_{0}$. For this optimum case, the propagation of SPPs can be extended up to approximately $10 \lambda_{0}$ compared with the case without cuboids. Moreover, it is shown that a subwavelength focus is achieved for all the cuboids, demonstrating that the propagation distance of SPPs can be extended without deteriorating the performance of PNJs.

\section{Design}

The chain of 3D dielectric cuboids considered in this work is schematically shown in Figure 1. It consists of an array of 6 cuboids with dimensions $d_{x} \times d_{y} \times d_{z}$ separated by a gap $p$ placed on top of a metal layer of gold. A dispersive model is used for gold following the Johnson and Christy experimental data [24]. The height of the metal layer is $100 \mathrm{~nm}$, i.e., well above the skin depth at $1550 \mathrm{~nm}$ which is below $30 \mathrm{~nm}$, so that it can be considered semi-infinite at the design wavelength [25]. The material of the cuboids is Silicon Nitride $\mathrm{Si}_{3} \mathrm{~N}_{4}$ with has a refractive index $n_{d}=1.97$. Note that a fixed value has been selected instead of a dispersive model to simplify the computation (this can be done because the variation of $n_{d}$ is very small—from 1.97 to 1.99 -within the spectral band of 750-2000 $\mathrm{nm}$ [23]). The whole structure is immersed in air with a refractive index $n_{0}=1$. With this configuration, the SPPs are excited from the back of the chain and propagate along the $z$-axis. The numerical simulations along this manuscript are done using the transient solver of the commercial software CST Microwave Studio ${ }^{\mathrm{TM}}$. As it is known, there are several techniques to excite SPPs, such as dipoles with different polarizations $[26,27]$. In our case we use a waveguide placed at the back of the structure with dimensions $5 \mu \mathrm{m} \times 0.15 \mu \mathrm{m}$ along the $x$ - and $z$-axis, respectively [23]. The total dimensions of the simulation space are $5 \mu \mathrm{m} \times 41 \mu \mathrm{m}$ along the $x$ - and $z$-axis, respectively. An extra air region of $1 \mu \mathrm{m}$ was added at the top of the structure and open boundary conditions were used for the entire simulation domain. With this condition, the air domain at the top of the structure is considered as semi-infinite, enabling to avoid undesirable reflections.
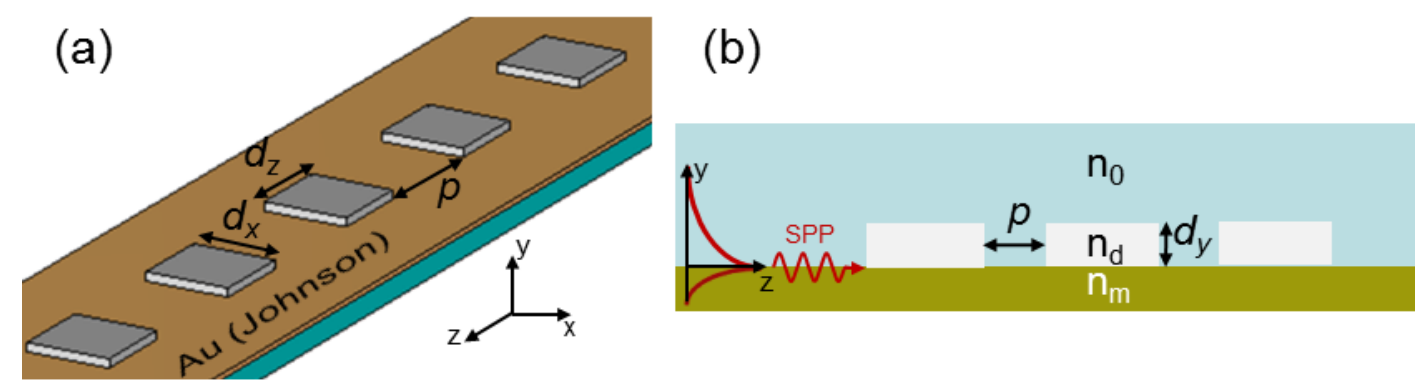

Figure 1. Schematic representation of the cuboid chain: (a) perspective and (b) lateral view. The dielectric cuboids have dimensions $d_{x} \times d_{y} \times d_{z}$ and are periodically placed along the $z$-axis with an air gap $p$ between them.

As it can be observed in Figure 1b, two different regions are recognized in the chain, namely, with and without cuboids, which correspond to an air-dielectric-metal and an air-metal multilayer structures, respectively. In both of them, the original multilayer problem can be reduced to an effective refractive index applying an homogenization technique, as described in [22,23]. 
Following this procedure, we find that the effective index of the region without cuboids is very similar to the index of air $(\sim 1.0043)$ at $1550 \mathrm{~nm}$, indicating that SPPs are not strongly coupled at the design wavelength. This is corroborated in the simulation results of Figure 2 where we have represented the $E_{y}$-field distribution on the metal surface. It can be observed that SPPs are weakly coupled to the surface of the metal and hence decay rapidly at $1550 \mathrm{~nm}$, reaching a short propagation length (i.e., distance at which the magnitude of the electric field decays by a factor $1 / e$ ) of $\sim 9 \lambda_{0}$, see green curve in Figure 3h.

Our aim in this work is to increase the propagation length by means of a chain of PNJ cuboids. In the next section we will perform a parametrical study to find the distance between cuboids as well as the cuboid height that optimizes the propagation length and the full-width at half-maximum along the transversal $x$-axis $\left(\mathrm{FWHM}_{\mathrm{x}}\right)$.

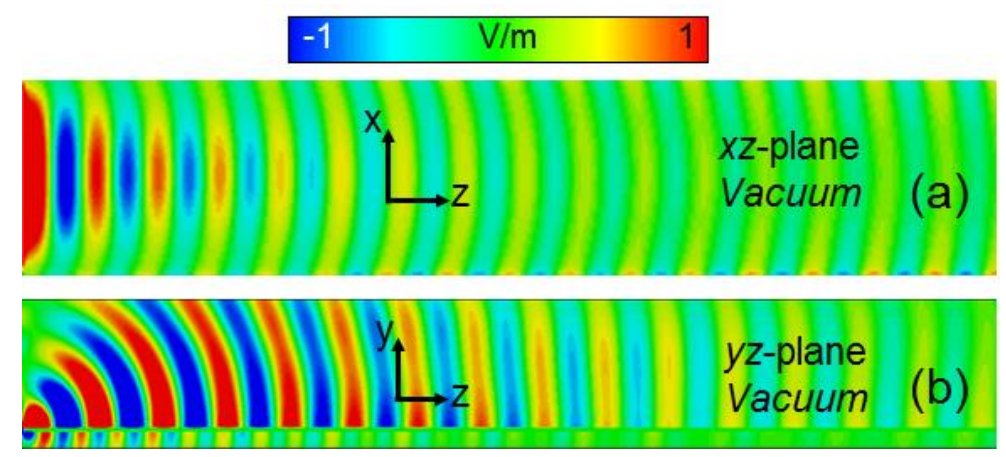

Figure 2. $E_{y}$-field distribution on the $x z$-plane (a) and (yz-plane) (b) for a flat gold film at $1550 \mathrm{~nm}$.

\section{Results and Discussion}

First, we evaluate the performance of the dielectric chain as the height of the 3D cuboids and the period $p$ between them are varied. From our previous works $[18,23]$ we know that the optimal lateral dimensions of the cuboids should be equal to the operation wavelength, $d_{x}=d_{z}=\lambda_{0}=1550 \mathrm{~nm}$ in order to produce the PNJ at the output face of the dielectric cuboids. With these dimensions, we evaluate the performance of the chain for $p$ ranging from 0 to $3 \lambda_{0}$ with a step of $0.5 \lambda_{0}$ and a height of cuboids $d_{y}$ varying from $80 \mathrm{~nm}$ to $240 \mathrm{~nm}$ with a step of $20 \mathrm{~nm}$. As it has been demonstrated in previous works, this dimension is critical in the performance of the PNJ, as it directly allows tuning the effective index of the region where the cuboid is present [22,23].

Contour plots showing the magnitude of the $E_{y}$ field at the design wavelength as a function of the propagation $z$-axis and height of the dielectric cuboids are shown in Figure $3 a-g$ for each value of $p$ under study. All the results have been normalized to the incident electric field amplitude at the back of the structure. This figure gives us all the information to evaluate the performance of the chain. Let us first study the influence of the period between the cuboids. As shown in the figure, the worst case is observed when $p=0$ where the decay of the SPPs is stronger compared with the other values of $p$. This is an expected result, as this period corresponds to the case when the cuboids are touching and hence only one PNJ is excited at the end of the structure $\left(z \sim 7 \lambda_{0}\right)$. As the cuboids are taken far apart, several peaks of high electric field concentration appear between cuboids. These peaks are the PNJs excited by each cuboid. The propagation length increases as $p$ is increased due to the PNJ excited by each cuboid produces an enhancement of the electric field at the focal length [23]. Note that this propagation length increases until it reaches a maximum at $p=2.5 \lambda_{0}$, and from there on it decreases again. The best case is when $p=2.5 \lambda_{0}$ and $d_{y}=120 \mathrm{~nm}$ (highlighted with a dashed white line in Figure $3 \mathrm{f}$ ). We have plotted in Figure $3 \mathrm{~h}$ the magnitude of the $E_{y}$ field along the propagation axis for this optimum case compared to a flat metal film. The SPP propagation length is $\sim 19 \lambda_{0}$, i.e., it is enhanced by a factor greater than two compared to the case without the cuboids (which reached only $\left.\sim 9 \lambda_{0}\right)$. 

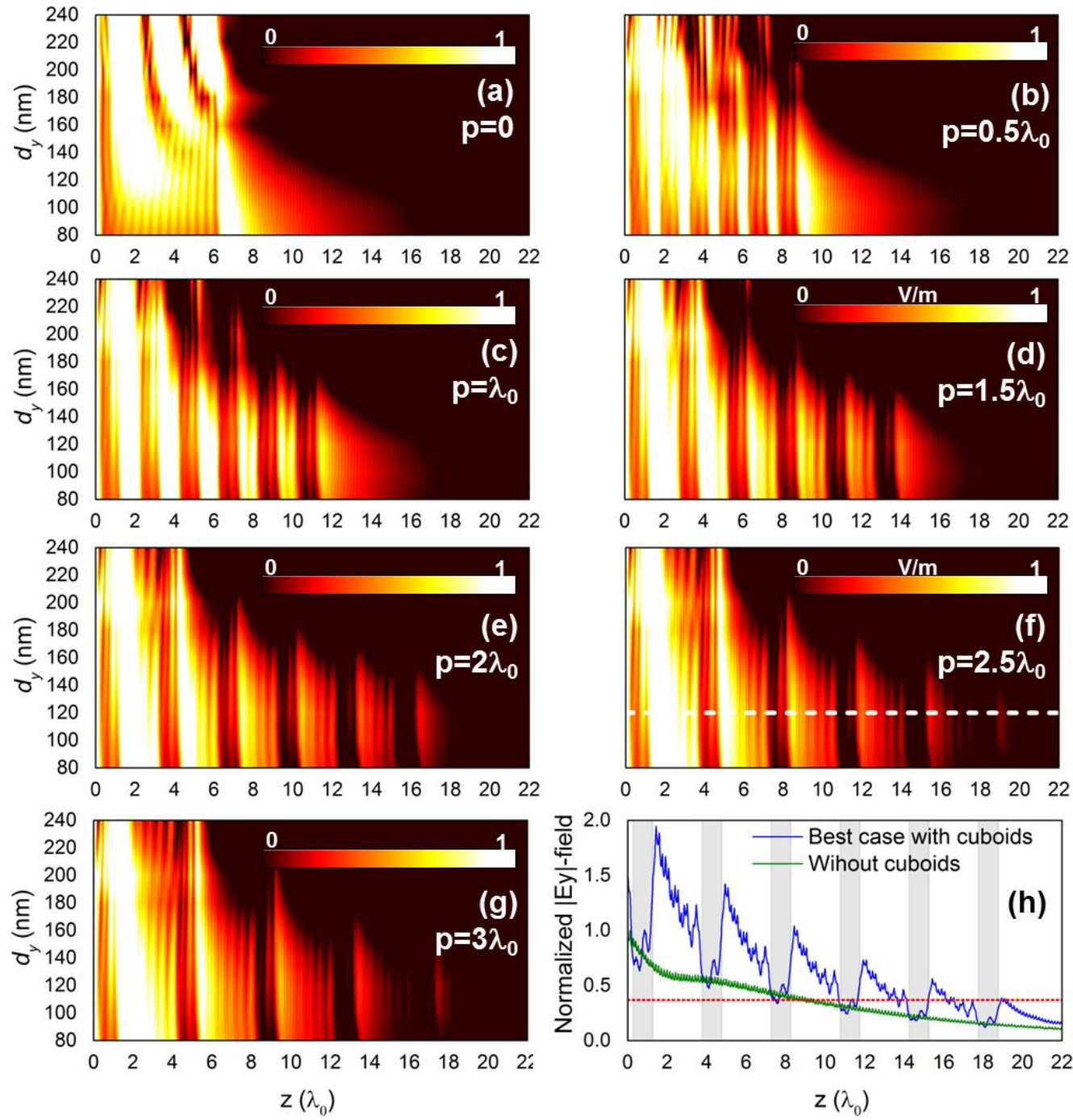

Figure 3. Normalized magnitude of the $E_{y}$ field as a function of the propagation $z$-axis and height $d_{y}$ of the cuboids when the distance between cuboids is: (a) $p=0$; (b) $p=0.5 \lambda_{0}$; (c) $p=\lambda_{0} ;$ (d) $p=1.5 \lambda_{0}$; (e) $p=2 \lambda_{0}$; (f) $p=2.5 \lambda_{0}$ and (g) $p=3 \lambda_{0}$. (h) Numerical results of the normalized magnitude of the electric field along the propagation axis at the surface of the metal for the optimal case, $p=2.5 \lambda_{0}$ and $d_{y}=120 \mathrm{~nm}$ (extracted from the dashed line shown in panel (f)) (blue curve) and a flat metal film (green curve). The red curve corresponds to a decay of 1 /e with respect to the amplitude of the incident surface plasmon polaritons (SPP) at the input. The 3D cuboids are also shown as grey blocks.

Regarding the height of the cuboids, it is interesting to observe that in all cases the longest SPPs propagation lengths are obtained for values of $d_{y}$ between approximately 100 and $140 \mathrm{~nm}$. This performance can be easily explained in terms of the effective index, $n_{e f f}$. For this analysis we concentrate on $p=2.5 \lambda_{0}$, which can be considered representative of all the studied cases. We have calculated the power distribution on the surface of the metal film for different values of $d_{y}$, see Figure 4a-e. Then we have extracted the propagation length for all the $d_{y}$ values considered and have plotted the results in Figure $4 \mathrm{f}$ along with the analytical values of the effective index contrast between the regions with and without cuboids [23]. For small height values $\left(d_{y}=80 ; 100 \mathrm{~nm}\right)$ the effective index contrast is close to 1 and hence, the PNJs are produced far away from the output surface of the cuboids $[18,23]$, giving as a result a poor illumination of the neighboring cuboid in the chain. On the other hand, for large height values $\left(d_{y}>120 \mathrm{~nm}\right)$ the index contrast increases as $d_{y}$ is increased. This implies that the PNJs are produced inside of the cuboids, as observed in Figure 4d,e 
and also discussed in $[18,23]$. Therefore, the excitation of consecutive cuboids is not efficient and the propagation length is reduced.

The longest propagation length is when $d_{y}=120 \mathrm{~nm}$, in which the effective index contrast is $\sim 1.2$. Note that with this value the PNJs are produced very near but slightly away from output surface of the cuboids (approximately at $0.16 \lambda_{0}$ ) as can be observed in Figure $4 \mathrm{~b}$. Since the PNJs are not just at the output surface of the cuboids, it allows obtaining the optimal illumination of the next cuboids of the chain. Note that this value of $d_{y}$ is different from the optimal case we found in our previous study [23]. Recall that in that paper the aim was to produce the PNJ just at the output surface of the cuboids and not to extend the SPP propagation. Anyway, it is interesting to highlight that the SPP propagation distance is enhanced for a wide range of values of $d_{y}$. This is an important feature in terms of the allowed tolerances for fabrication purposes. If we take $120 \mathrm{~nm}$ as a reference, the height of the cuboids may be fabricated with a tolerance of $\pm 40 \mathrm{~nm}$ without strongly altering the SPP propagation distance.
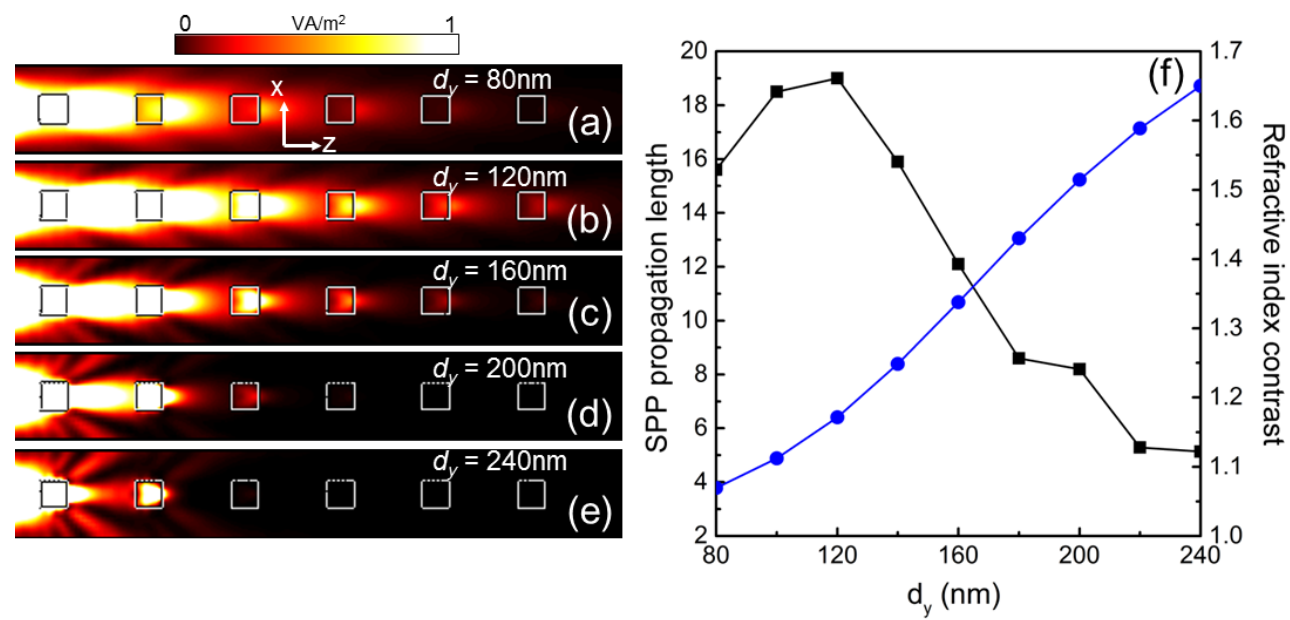

Figure 4. Power distribution on the $x z$-plane for the case of 3D dielectric cuboids placed periodically with an air gap of $p=2.5 \lambda_{0}$ and height: (a) $d_{y}=80 \mathrm{~nm}$; (b) $d_{y}=120 \mathrm{~nm}$; (c) $d_{y}=160 \mathrm{~nm}$; (d) $d_{y}=200 \mathrm{~nm}$ and (e) $d_{y}=240 \mathrm{~nm}$. (f) Numerical results of SPP propagation distance (black curve) and index contrast (blue curve) as a function of the cuboid height.

For the sake of completeness, we also evaluate the PNJ performance in terms of the transversal resolution. We have represented in Figure 5 the full-width at half-maximum along the $x$-axis $\left(F W H M_{x}\right.$, defined at the distance along the $x$-axis at which the power decays to half of its maximum) of the PNJs produced by all the cuboids in the array, for the optimal case under study. It can be observed that a subwavelength focus is obtained in all cases with a resolution below $0.75 \lambda_{0}$.

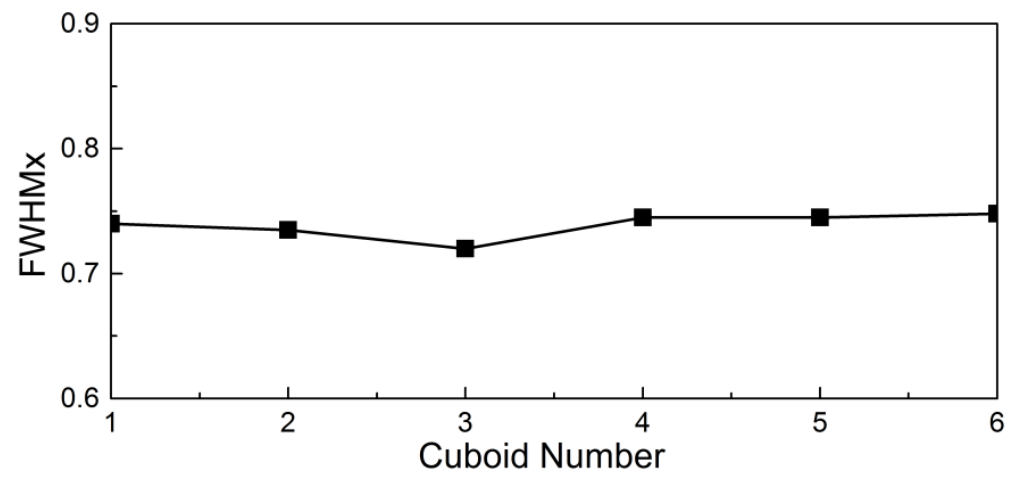

Figure 5. Numerical results of the full-width at half-maximum along the transversal $x$-axis of the photonic nanojets (PNJ) produced by each cuboid for the optimal case when $p=2.5 \lambda_{0}$ and $d_{y}=120 \mathrm{~nm}$. 


\section{Conclusions}

In this work, a dielectric chain consisting of periodically spaced 3D dielectric cuboids has been thoroughly studied, with the aim to increase the propagation distance of SPPs at the telecom wavelength of $1550 \mathrm{~nm}$. It has been shown that the propagation distance can be increased by a factor greater than two (up to $\sim 19 \lambda_{0}$ ) for the optimal case when the gap between them is $2.5 \lambda_{0}$ and the height of the cuboids is $140 \mathrm{~nm}$. It has been shown that for heights $>180 \mathrm{~nm}$, the propagation distance of the SPPs is strongly reduced which is a direct consequence of the ratio between the effective refractive indexes of the air-metal-dielectric and air-metal regions of the structure. Moreover, it has been demonstrated that a subwavelength transversal resolution of the PNJs can be achieved at the output of each cuboid of the array. The results here presented may be useful in the design of different photonic applications such as beam steerers and SPP optical tweezers, which are now under study.

Acknowledgments: This work was supported by the Spanish Ministerio de Economía y Competitividad under contract TEC2014-51902-C2-2-R and partially was supported by the Mendeleev scientific fund of Tomsk State University No 8.2.48.2015. V.P.-P. is sponsored by Spanish Ministerio de Educación, Cultura y Deporte under grant FPU AP-2012-3796. M.B. is sponsored by the Spanish Government via RYC-2011-08221.

Author Contributions: V.P.-P. and M.B. designed and studied the idea of using 3D dielectric cuboids to increase the propagation of SPPs at the design wavelength. Also, V.P.-P. and M.B. performed the numerical evaluation of the structures and wrote the paper. I.V.M. and O.V.M. helped in the discussion and analysis of the results here presented.

Conflicts of Interest: The authors declare no conflict of interest.

\section{Abbreviations}

The following abbreviations are used in this manuscript:

PNJ Photonic Nanojet

SPP Surface Plasmon

$\mathrm{FWHM}_{\mathrm{x}} \quad$ Full-width at half-maximum along the transversal $x$-axis

\section{References}

1. Born, M.; Wolf, E. Principles of Optics, 7th ed.; Cambridge University Press: New York, NY, USA, 1999.

2. Pendry, J.B. Negative refraction makes a perfect lens. Phys. Rev. Lett. 2000, 85, 3966-3969. [CrossRef] [PubMed]

3. Grbic, A.; Eleftheriades, G.V. Overcoming the Diffraction Limit with a Planar Left-Handed Transmission-Line Lens. Phys. Rev. Lett. 2004, 92, 117401-117403. [CrossRef] [PubMed]

4. Jacob, Z.; Alekseyev, L.V.; Narimanov, E. Optical Hyperlens: Far-field imaging beyond the diffraction limit. Opt. Express 2006, 14, 8247-8256. [CrossRef] [PubMed]

5. Liu, Z.; Lee, H.; Xiong, Y.; Sun, C.; Zhang, X. Far-field optical hyperlens magnifying sub-diffraction-limited objects. Science 2007, 315, 1686. [CrossRef] [PubMed]

6. Huang, F.M.; Zheludev, N.I. Super-resolution without evanescent waves. Nano Lett. 2009, 9, $1249-1254$. [CrossRef] [PubMed]

7. Rogers, E.T.F.; Lindberg, J.; Roy, T.; Savo, S.; Chad, J.E.; Dennis, M.R.; Zheludev, N.I. A super-oscillatory lens optical microscope for subwavelength imaging. Nat. Mater. 2012, 11, 432-435. [CrossRef] [PubMed]

8. Wang, Z.; Guo, W.; Li, L.; Luk'yanchuk, B.; Khan, A.; Liu, Z.; Chen, Z.; Hong, M. Optical virtual imaging at $50 \mathrm{~nm}$ lateral resolution with a white-light nanoscope. Nat. Commun. 2011, 2, 218. [CrossRef] [PubMed]

9. Hao, X.; Kuang, C.; Liu, X.; Zhang, H.; Li, Y. Microsphere based microscope with optical super-resolution capability. Appl. Phys. Lett. 2011, 99, 203102. [CrossRef]

10. Li, X.; Chen, Z.; Taflove, A.; Backman, V. Optical analysis of nanoparticles via enhanced backscattering facilitated by 3-D photonic nanojets. Opt. Express 2005, 13, 526-533. [CrossRef] [PubMed]

11. Heifetz, A.; Huang, K.; Sahakian, A.V.; Li, X.; Taflove, A.; Backman, V. Experimental confirmation of backscattering enhancement induced by a photonic jet. Appl. Phys. Lett. 2006, 89, 221118. [CrossRef] 
12. Heifetz, A.; Kong, S.-C.; Sahakian, A.V.; Taflove, A.; Backman, V. Photonic Nanojets. J. Comput. Theor. Nanosci. 2009, 6, 1979-1992. [CrossRef] [PubMed]

13. Liu, C.; Chang, L.; Yang, L. Photonic Nanojet in Non-spherical Micro-particles. In Proceedings of the 9th IEEE International Conference on Nano/Micro Engineered and Molecular Systems (NEMS), Waikiki Beach, HI, USA, 13-16 April 2014; pp. 536-539.

14. Mao, X.; Yang, Y.; Dai, H.; Luo, D.; Yao, B.; Yan, S. Tunable photonic nanojet formed by generalized Luneburg lens. Opt. Express 2015, 23, 26426. [CrossRef] [PubMed]

15. Smolyaninova, V.; Lahneman, D.; Adams, T.; Gresock, T.; Zander, K.; Jensen, C.; Smolyaninov, I. Experimental Demonstration of Luneburg Waveguides. Photonics 2015, 2, 440-448. [CrossRef]

16. Liu, C. Ultra-elongayed photonic nanojets generated by a graded-index microellipsoid. Prog. Electromagn. Res. Lett. 2013, 37, 153-165. [CrossRef]

17. Born, B.; Krupa, J.D.; Geoffroy-Gagnon, S.; Holzman, J.F. Integration of photonic nanojets and semiconductor nanoparticles for enhanced all-optical switching. Nat. Commun. 2015, 6, 8097. [CrossRef] [PubMed]

18. Pacheco-Peña, V.; Beruete, M.; Minin, I.V.; Minin, O.V. Terajets produced by dielectric cuboids. Appl. Phys. Lett. 2014, 105, 084102. [CrossRef]

19. Pacheco-Peña, V.; Beruete, M.; Minin, I.V.; Minin, O.V. Multifrequency focusing and wide angular scanning of terajets. Opt. Lett. 2015, 40, 245-248. [CrossRef] [PubMed]

20. Minin, I.V.; Minin, O.V.; Pacheco-Peña, V.; Beruete, M. Localized photonic jets from flat, three-dimensional dielectric cuboids in the reflection mode. Opt. Lett. 2015, 40, 2329-2332. [CrossRef] [PubMed]

21. Minin, I.V.; Minin, O.V.; Pacheco-Peña, V.; Beruete, M. All-dielectric periodic terajet waveguide using an array of coupled cuboids. Appl. Phys. Lett. 2015, 106, 254102. [CrossRef]

22. Ju, D.; Pei, H.; Jiang, Y.; Sun, X. Controllable and enhanced nanojet effects excited by surface plasmon polariton. Appl. Phys. Lett. 2013, 102, 171109. [CrossRef]

23. Pacheco-Peña, V.; Minin, I.V.; Minin, O.V.; Beruete, M. Backscattering enhancement of small particles through Photonic Nanojets excited by Surface Plasmons at $1550 \mathrm{~nm}$. Phys. Rev. B 2015. under review.

24. Johnson, P.B.; Christry, R.W. Optical Constants of the Noble Metals. Phys. Rev. B 1972, 6, 4370-4379. [CrossRef]

25. Burke, J.J.; Stegeman, G.I.; Tamir, T. Surface-polariton-like waves guided by thin, lossy metal films. Phys. Rev. B 1986, 33, 5186-5201. [CrossRef]

26. Fortuño, F.J.R.; Marino, G.; Ginzburg, P.; Connor, D.O.; Wurtz, G.; Martinez Abietar, A.; Zayats, A. Near-Field Interference for the Unidirectional Excitation of Electromagnetic Guided Modes. Science 2013, 340, 328-330. [CrossRef] [PubMed]

27. Mueller, J.P.B.; Capasso, F. Asymmetric surface plasmon polariton emission by a dipole emitter near a metal surface. Phys. Rev. B Condens. Matter Mater. Phys. 2013, 88, 1-6. [CrossRef]

(C) 2016 by the authors; licensee MDPI, Basel, Switzerland. This article is an open access article distributed under the terms and conditions of the Creative Commons by Attribution (CC-BY) license (http://creativecommons.org/licenses/by/4.0/). 